\title{
Efficacy of Fungicidal Seed Treatments on Seed Borne Diseases of Green Gram
}

\author{
A. J. Deshmukh ${ }^{1 *}$, A. N. Sabalpara ${ }^{2}$ and R. P. Bambharolia ${ }^{1}$
}

${ }^{1}$ Navsari Agricultural University, College of Agriculture, Waghai, Gujarat (394 730), India

${ }^{2}$ Navsari Agricultural University, Navsari, Gujarat (396 450), India

\section{Corresponding Author}

A. J. Deshmukh

e-mail: amol_deshmukhnau@nau.in

\author{
Article History \\ Article ID: IJEP00378 \\ Received in 05 ${ }^{\text {th }}$ July, 2020 \\ Received in revised form $21^{\text {st }}$ July, 2020 \\ Accepted in final form $29^{\text {th }}$ July, 2020
}

\begin{abstract}
The experiment was conducted to perceive the effect of fungicidal seed treatment on seed borne diseases of green gram on susceptible cv. GM-3 with nine different fungicides. Results revealed that dry seed treatment with either mix formulation of carbendazim + mancozeb or thiophanate methyl or carbendazim @ $2.5 \mathrm{~g} \mathrm{~kg}^{-1}$ seeds is very effective in field to control Macrophomina leaf blight, Alternaria leaf spot and Anthracnose diseases. Two years pooled data indicated that PDI of Macrophomina leaf bilght was significantly lower in dry seed treatment with carbendazim + mancozeb $(0.99 \%, 12.90 \%)$ which was statistically at par with thiophanate methyl $(1.54 \%, 13.80 \%)$ followed by carbendazim $(0.99 \%, 14.06 \%)$ at 35 and 65 DAS, respectively. Two years pooled data of Alternaria leaf spot indicated that the PDI of was significantly lower in dry seed treatment with carbendazim + mancozeb $(2.64 \%, 11.15 \%)$ which was statistically at par with thiophanate methyl $(2.74 \%, 11.30 \%)$ followed by carbendazim $(2.84 \%, 11.37 \%)$ at 35 and 65 DAS. Two years pooled data of Anthracnose disease indicated that the PDI of Anthracnose was significantly lower in dry seed treatment with carbendazim + mancozeb $(0.81 \%, 6.71 \%)$.
\end{abstract}

Keywords: Green gram, seed treatment, seed borne diseases

\section{Introduction}

Greengram [Vigna radiata (L.) Wilczek] is an important pulse crop locally known as mung bean, golden gram, mung or moong (John, 1991). It is considered to be native of India and Central Asia and grown widely in India, Pakistan, Sri Lanka, Thailand, Laos, Combodia, Vietnam, Indonesia, Malaysia, South China, Formosa, Phillipines, Taiwan etc. It is also grown to lesser extent in many parts of the Africa, USA and Australia (Agrawal, 1989). In India, green gram occupies an area of 3.34 million hectares with the total production of 1.06 million tones and productivity of $415 \mathrm{~kg} \mathrm{ha}^{-1}$. In India, major green gram growing states are Andhra Pradesh, Madhya Pradesh, Orissa, Maharashtra, Rajasthan, Bihar and Gujarat. In Gujarat, it is cultivated on about 2.09 lakh hectares with total production of 1.14 lakh tones and productivity of $546 \mathrm{~kg} \mathrm{ha}^{-1}$ during Kharif season. India is considered as the homeland of most of the grain legumes and the lion share of protein is supplied by pulses to majority of Indians. Pulses are the integral part of Indian dietary system due to their richness in protein and other important nutrients. They are said to be poor man's meat and rich man's vegetables. As per recommendation of $\mathrm{WHO}$, minimum requirement of pulses is $80 \mathrm{gm} \mathrm{capita}^{-1}$ day $^{-}$ 1. Green gram is an important pulse crop used primarily for food. The dried seeds may be eaten as whole or split, cooked or fermented, or parched, milled and ground into flour. Whole or split, they are used to make dal, soups and curries and are added to various spiced and fried dishes. Popular fermented foods in India, such as idli and dosa, are made from dough of rice and green gram together. The flour is used to make noodles, breads and biscuits. An important green gram by product is starch noodle, which is transparent, easy to cook and sores well (Summerfield and Roberts, 1985). Green gram seeds can be germinated to produce bean sprouts, the nutritional value of which is comparable with asparagus or mushrooms (Tan, 1973). The composition of green gram seed is approximately 25.0 to $28.0 \%$ protein, 1.0 to $1.5 \%$ oil, 3.5 to $4.5 \%$ fiber, 4.5 to $5.5 \%$ ash and 62.0 to $65.0 \%$ carbohydrate on a dry weight basis (Singh et al., 1970; Tsou et al., 1979). Disease is the major constraint in economic crop production as they inflict heavy losses. Like other crops, green gram is also attacked by many diseases during seed germination to seed production and maturity. Over 35 fungal pathogens, few viral, bacterial and nematode species are known to attack green gram resulting into substantial yield losses (Agrawal, 1989). Among these, seed borne fungal diseases are important in reducing the yield and seed quality of green gram (Sinha and Prasad, 1981). Most of the fungal diseases such as anthracnose, leaf spots, leaf blights and root rot causing severe losses are seed borne in nature in green gram. Hence, 
seeds of moong have been reported to play an important role in the dissemination of various pathogens (Rangaswami and Prasad, 1960; Rayen, 1961). These fungi affect developing seeds adversely such as seed discoloration, molding of seed surface, endosperm degradation and reduced seed filling resulting in seed deformalities such as shriveling of seeds as well as small seed formation. The seed infecting fungi not only damages the quantity but also quality of seed. Similarly, nutritional status of green gram seeds is likely to be hampered by depleting the protein content and by the disturbance of the reducing sugar as well as starch content due to seed mycoflora (Bilgrami, et al. 1978). To increase the production of green gram qualitatively and quantitatively, farmer requires healthy quality seeds with high percentage of germination and purity. Hence, it is imperative that the seeds must be tested before they are sown in the field. Another adverse effect of seed borne pathogens is that it will contaminate the areas which were disease free previously. Hence, it necessitates the eradication of seed borne inoculum through various seed treatments and through the enforcement of proper domestic and international quarantine acts and procedures. Degree of damage to the green gram seeds is influenced by various biotic and abiotic factors along with virulent pathogen, varietal susceptibility and prevailing humidity particularly during developing pod-stage and harvesting time etc. Seed treatment is the oldest practice in plant protection and now, this is an attractive delivery system with either fungicide of bioprotectants. As seed treatments provide economical and relatively non-polluting delivery systems for protective materials compared to other field application systems. Fungicides applied to seeds may not only protect seeds from seed borne diseases but also protect roots and increase the plant growth indirectly. Patel (2003) and Tandel (2004) studied leaf spot ( $A$. alternata) and leaf blight ( $M$. phaseolina) of green gram and found that both the diseases were seed borne in nature and cause heavy losses in green gram. Hence, the present investigation was conducted to perceive the effect of fungicidal seed treatment to control the seed borne diseases with nine different fungicides in green gram Cv. GM-3.

\section{Materials and Methods}

A field trial was conducted for two seasons at N.M. College of Agriculture, Research Farm, N.A.U., Navsari. Agro climatic sub region was South Gujarat heavy rainfall zone; variety was GM3. Seeds collected from Pulse Research Station, N.A.U., Navsari were taken and sown after dry seed treatment with different fungicides viz., Carbendazim $\left(2.5 \mathrm{~g} \mathrm{~kg}^{-1}\right)$, Kasugomycin $(2.5 \mathrm{~g}$ $\left.\mathrm{kg}^{-1}\right)$, Thiram $\left(3.5 \mathrm{~g} \mathrm{~kg}^{-1}\right)$, Captan $\left(3.5 \mathrm{~g} \mathrm{~kg}^{-1}\right)$, Carbendazim + mancozeb (2.5 g kg $)$, MEMC (2.5 $\left.\mathrm{g} \mathrm{kg}^{-1}\right)$, Mancozeb (3.5 $\left.\mathrm{g} \mathrm{kg}^{-1}\right)$, Thiophanate methyl $\left(3.5 \mathrm{~g} \mathrm{~kg}^{-1}\right)$ and Difenconazole $\left(2.5 \mathrm{~g} \mathrm{~kg}^{-1}\right)$. Recommended agronomic practices like seed rate, spacing, irrigation, fertilizer application and intercultural operations were followed during whole experiment. Three replication of each treatment of individual plot size of $3 \times 3 \mathrm{~m}^{2}$. of having spacing $30 \times 10 \mathrm{~cm}^{2}$ were maintained. The whole experimental area was $36.5 \times 13 \mathrm{~m}^{2}$.

The observations on Percent Disease Intensity (PDI) of three diseases viz., Macrophomina leaf blight, Alternaria leaf spot and anthracnose observed in the field were recorded at 35 DAS and 65 DAS by selecting 10 plants plot $^{-1}$ in each treatment by observing three trifoliate leaves first of base, second of middle and third of upper portion of the selected plant starting from the initiation up to harvest each at 15 days interval of the crop using standard graded scale and data thus obtained was analyzed with RBD design and tabulated separately for each disease. Observations with regard to infection and symptoms development of each disease were recorded on the basis different disease grading scale. The rating scale for Macrophomina leaf blight was 0-6 (Kumar et al. 1969) as 0 -leaves disease free, 1 -leaves area covered up to $5 \%$, 2-leaves area covered 6-10\% , 3-leaves area covered $11-25 \%$, 4-leaves area covered 26-50\%, 5-leaves area covered 51-75\%, 6 -leaves area covered $76-100 \%$. The rating scale for Alternaria leaf spot was0-5 (Mathur et al., 1972) as 0-no infection, 1-1 to $20 \%$ area infected, $2-21$ to $40 \%$ area infected, $3-41$ to $60 \%$ area infected, 4-61 to $80 \%$ area infected and 5-above $80 \%$ area infected. The rating scale for anthracnose was 0-5 (Rajkumar and Mukhopadhyay, 1986) as 0-no infection, 1-disease infection trace, 2-1-25\% infection, 3-26-50\% infection, 4- 51$75 \%$ infection, 5-76\% and more infection.

Formula for calculating disease intensity (\%):

$\mathrm{PDI}=(\Sigma$ of ratings of infected leaves observed/No. of leaves observed $\times$ Maximum disease score) $\times 100$

\section{Results and Discussion}

The occurrence of three different diseases viz., Macrophomina leaf blight, Alternaria leaf spot and anthracnose (C. capsici) was reported in the field and data thus obtained was recorded and tabulated (Table 1, 2 and 3).

\subsection{Macrophomina leaf blight at 35 DAS}

The pooled data of two years (Table 1 ) indicated that the PDI of Macrophomina leaf blight was significantly reduced in all the treatments over the control at 35 DAS. It was significantly lower in dry seed treatment with carbendazim + mancozeb (0.99\%) as compared to the rest but was statistically at par with thiophanate methyl (1.54\%). Next best in order of merit was carbendazim (1.87\%). Comparatively higher leaf blight was found in captan (2.41\%) followed by thiram $(2.33 \%)$, difenconazole (2.53\%) and MEMC (2.58\%). Mancozeb (2.86\%) and kasugomycin (3.42\%) were comparatively poor in their efficay. The highest disease was recorded in the control $(8.67 \%)$. Maximum Macrophomina leaf blight control was recorded in carbendazim + mancozeb (88.58\%) followed by thiophanate methyl (82.24\%), carbendazim (78.43\%), captan (73.13\%), thiram (72.20\%), difenconazole (70.82\%), MEMC (70.24\%), mancozeb (67.01\%) and kasugomycin $(60.55 \%)$ treatment. 


\begin{tabular}{|c|c|c|c|c|c|c|c|c|c|}
\hline \multirow{3}{*}{$\begin{array}{l}\text { Sr. } \\
\text { No. }\end{array}$} & \multirow[t]{3}{*}{ Treatment } & \multicolumn{8}{|c|}{ PDI (Leaf blight: M. phaseolina) } \\
\hline & & \multicolumn{4}{|c|}{35 DAS } & \multicolumn{4}{|c|}{65 DAS } \\
\hline & & $\begin{array}{l}\text { First } \\
\text { year }\end{array}$ & $\begin{array}{l}\text { Second } \\
\text { year }\end{array}$ & Pooled & PDC & $\begin{array}{l}\text { First } \\
\text { year }\end{array}$ & $\begin{array}{c}\text { Second } \\
\text { year }\end{array}$ & Pooled & PDC \\
\hline 1. & Carbendazim (Bavistin 50\% WP) & $\begin{array}{c}8.20 \\
(2.04)\end{array}$ & $\begin{array}{c}7.50 \\
(1.70)\end{array}$ & $\begin{array}{c}7.86 \\
(1.87)\end{array}$ & 78.43 & $\begin{array}{c}22.40 \\
(14.52)\end{array}$ & $\begin{array}{c}21.65 \\
(13.61)\end{array}$ & $\begin{array}{c}22.02 \\
(14.06)\end{array}$ & 54.62 \\
\hline 2. & Kasugomycin (Kasugomycin 12\% WP) & $\begin{array}{l}10.92 \\
(3.59)\end{array}$ & $\begin{array}{l}10.40 \\
(3.26)\end{array}$ & $\begin{array}{l}10.66 \\
(3.42)\end{array}$ & 60.55 & $\begin{array}{c}25.37 \\
(18.36)\end{array}$ & $\begin{array}{c}24.75 \\
(17.53)\end{array}$ & $\begin{array}{c}25.06 \\
(17.94)\end{array}$ & 42.09 \\
\hline 3. & Thiram (Thirum 75\% WP) & $\begin{array}{c}8.45 \\
(2.16)\end{array}$ & $\begin{array}{c}9.08 \\
(2.49)\end{array}$ & $\begin{array}{c}8.77 \\
(2.33)\end{array}$ & 73.13 & $\begin{array}{c}23.71 \\
(16.17)\end{array}$ & $\begin{array}{c}23.02 \\
(15.29)\end{array}$ & $\begin{array}{c}23.37 \\
(15.73)\end{array}$ & 49.23 \\
\hline 4. & Captan (Captan 75\% WP) & $\begin{array}{c}9.23 \\
(2.57)\end{array}$ & $\begin{array}{c}8.61 \\
(2.24)\end{array}$ & $\begin{array}{c}8.92 \\
(2.41)\end{array}$ & 72.20 & $\begin{array}{c}22.81 \\
(15.03)\end{array}$ & $\begin{array}{c}22.08 \\
(14.13)\end{array}$ & $\begin{array}{c}22.45 \\
(14.58)\end{array}$ & 52.94 \\
\hline 5. & Carbendazim+mancozeb (Sixer 75\% WP) & $\begin{array}{c}6.18 \\
(1.16)\end{array}$ & $\begin{array}{c}5.22 \\
(0.83)\end{array}$ & $\begin{array}{c}5.72 \\
(0.99)\end{array}$ & 88.58 & $\begin{array}{c}21.44 \\
(13.37)\end{array}$ & $\begin{array}{c}20.65 \\
(12.43)\end{array}$ & $\begin{array}{c}21.05 \\
(12.90)\end{array}$ & 58.36 \\
\hline 6. & MEMC (Emisan 6\% WP) & $\begin{array}{c}9.54 \\
(2.75)\end{array}$ & $\begin{array}{c}8.93 \\
(2.41)\end{array}$ & $\begin{array}{c}9.24 \\
(2.58)\end{array}$ & 70.24 & $\begin{array}{c}24.04 \\
(16.59)\end{array}$ & $\begin{array}{c}23.36 \\
(15.72)\end{array}$ & $\begin{array}{c}23.70 \\
(16.16)\end{array}$ & 47.84 \\
\hline 7. & Mancozeb (Diathane M-45, 75\% WP) & $\begin{array}{l}10.02 \\
(3.03)\end{array}$ & $\begin{array}{c}9.44 \\
(2.69)\end{array}$ & $\begin{array}{c}9.73 \\
(2.86)\end{array}$ & 67.01 & $\begin{array}{c}24.68 \\
(17.44)\end{array}$ & $\begin{array}{c}24.03 \\
(16.59)\end{array}$ & $\begin{array}{c}24.36 \\
(17.01)\end{array}$ & 45.09 \\
\hline 8. & Thiophanate methyl (Topsin M-75\% WP) & $\begin{array}{c}7.52 \\
(1.71)\end{array}$ & $\begin{array}{c}6.74 \\
(1.38)\end{array}$ & $\begin{array}{c}7.14 \\
(1.54)\end{array}$ & 82.24 & $\begin{array}{c}22.18 \\
(14.25)\end{array}$ & $\begin{array}{c}21.42 \\
(13.34)\end{array}$ & $\begin{array}{c}21.80 \\
(13.80)\end{array}$ & 55.46 \\
\hline 9. & Difenconazole (Score 25\% EC) & $\begin{array}{c}9.46 \\
(2.70)\end{array}$ & $\begin{array}{l}8.85 \\
(2.37)\end{array}$ & $\begin{array}{c}9.16 \\
(2.53)\end{array}$ & 70.82 & $\begin{array}{c}23.86 \\
(16.36)\end{array}$ & $\begin{array}{c}23.17 \\
(15.48)\end{array}$ & $\begin{array}{c}23.52 \\
(15.92)\end{array}$ & 48.61 \\
\hline 10. & Control (without treatment) & $\begin{array}{c}17.30 \\
(8.84)\end{array}$ & $\begin{array}{l}16.96 \\
(8.51)\end{array}$ & $\begin{array}{l}17.13 \\
(8.67)\end{array}$ & & $\begin{array}{c}33.88 \\
(31.07)\end{array}$ & $\begin{array}{c}33.77 \\
(30.90)\end{array}$ & $\begin{array}{c}33.82 \\
(30.98)\end{array}$ & - \\
\hline \multicolumn{2}{|c|}{ SEm \pm} & 0.75 & 0.82 & 0.50 & - & 1.05 & 1.10 & 0.68 & - \\
\hline \multicolumn{2}{|c|}{$\mathrm{CD}(p=0.05)$} & 2.22 & 2.43 & 1.45 & - & 3.22 & 3.38 & 1.98 & - \\
\hline \multicolumn{2}{|c|}{ C.V. \% } & 13.46 & 15.69 & 14.56 & - & 8.65 & 8.60 & 8.62 & - \\
\hline
\end{tabular}

Data outside the paranthesis is ARCSIN transformed data, PDI-Percent disease intensity, PDC-Percent disease control

\subsection{Macrophomina leaf blight at 65 DAS}

The pooled data of two years (Table-1) indicated that the PDI of Macrophomina leaf blight was significantly reduced in all the treatments over the control at 65 DAS. It was significantly lower in dry seed treatment with carbendazim + mancozeb $(12.90 \%)$ as compared to the rest but was statistically at par with thiophanate methyl (13.80\%), carbendazim $(14.06 \%)$ and captan (14.58\%). Next best in order to merit was thiram (15.73\%) followed by difenconazole (15.92\%), MEMC (16.16\%), mancozeb (17.01\%) and kasugomycin (17.94\%). Maximum control of Macrophomina leaf blight was recorded in carbendazim + mancozeb (58.36\%) followed by thiophanate methyl (55.46\%), carbendazim (54.62\%), captan $(52.94 \%)$, thiram (49.23\%), difenconazole (48.61\%), MEMC (47.84\%), mancozeb (45.09\%) and kasugomycin (42.09\%).

\subsection{Alternaria leaf spot at 35 DAS}

The pooled data of two years (Table 2 ) indicated that the PDI of Alternaria leaf spot was significantly reduced in all the treatments over the control at 35 DAS. It was significantly lower in dry seed treatment with carbendazim + mancozeb (2.64\%) as compared to the rest but was statistically at par with thiophanate methyl $(2.74 \%)$, carbendazim $(2.84 \%)$ and captan (2.94\%). Next best in order to merit was thiram (4.75\%) followed by difenconazole (5.05\%), MEMC (5.05\%), mancozeb (5.79\%) and kasugomycin (5.87\%). The highest disease was recorded in the control (10.97\%). Maximum control of Alternaria leaf spot was recorded in carbendazim + mancozeb (75.93\%) followed by thiophanate methyl (75.02\%), carbendazim $(74.11 \%)$, captan $(73.20 \%)$, thiram $(56.70 \%)$, difenconazole (53.97\%), MEMC (53.97\%), mancozeb (47.22\%) and kasugomycin (46.49\%).

\subsection{Alternaria leaf spot at 65 DAS}

The pooled data of two years (Table 2 ) indicated that the PDI of Alternaria leaf spot was significantly reduced in all the treatments over the control at 65 DAS. It was significantly lower in dry seed treatment with carbendazim + mancozeb $(11.15 \%)$ as compared to the rest but was statistically at par 


\begin{tabular}{|c|c|c|c|c|c|c|c|c|c|}
\hline \multirow{3}{*}{$\begin{array}{l}\text { Sr. } \\
\text { No. }\end{array}$} & \multirow[t]{3}{*}{ Treatment } & \multicolumn{8}{|c|}{ PDI (Leaf spot: A. alternata) } \\
\hline & & \multicolumn{4}{|c|}{35 DAS } & \multicolumn{4}{|c|}{65 DAS } \\
\hline & & $\begin{array}{l}\text { First } \\
\text { year }\end{array}$ & $\begin{array}{l}\text { Second } \\
\text { year }\end{array}$ & Pooled & PDC & $\begin{array}{l}\text { First } \\
\text { year }\end{array}$ & $\begin{array}{c}\text { Second } \\
\text { year }\end{array}$ & Pooled & PDC \\
\hline 1. & Carbendazim (Bavistin 50\% WP) & $\begin{array}{c}9.76 \\
(2.88)\end{array}$ & $\begin{array}{c}9.64 \\
(2.81)\end{array}$ & $\begin{array}{c}9.70 \\
(2.84)\end{array}$ & 74.11 & $\begin{array}{c}19.68 \\
(11.34)\end{array}$ & $\begin{array}{c}19.74 \\
(11.41)\end{array}$ & $\begin{array}{c}19.71 \\
(11.37)\end{array}$ & 59.47 \\
\hline 2. & Kasugomycin (Kasugomycin 12\% WP) & $\begin{array}{l}14.04 \\
(5.89)\end{array}$ & $\begin{array}{l}13.99 \\
(5.85)\end{array}$ & $\begin{array}{l}14.02 \\
(5.87)\end{array}$ & 46.49 & $\begin{array}{c}22.34 \\
(14.45)\end{array}$ & $\begin{array}{c}22.42 \\
(14.55)\end{array}$ & $\begin{array}{c}22.38 \\
(14.50)\end{array}$ & 48.31 \\
\hline 3. & Thiram (Thirum 75\% WP) & $\begin{array}{l}12.63 \\
(4.78)\end{array}$ & $\begin{array}{l}12.56 \\
(4.73)\end{array}$ & $\begin{array}{l}12.59 \\
(4.75)\end{array}$ & 56.70 & $\begin{array}{c}21.48 \\
(13.41)\end{array}$ & $\begin{array}{c}21.56 \\
(13.50)\end{array}$ & $\begin{array}{c}21.52 \\
(13.46)\end{array}$ & 52.01 \\
\hline 4. & Captan (Captan 75\% WP) & $\begin{array}{c}9.93 \\
(2.98)\end{array}$ & $\begin{array}{c}9.81 \\
(2.90)\end{array}$ & $\begin{array}{c}9.87 \\
(2.94)\end{array}$ & 73.20 & $\begin{array}{c}19.74 \\
(11.41)\end{array}$ & $\begin{array}{c}19.81 \\
(11.48)\end{array}$ & $\begin{array}{c}19.77 \\
(11.45)\end{array}$ & 59.18 \\
\hline 5. & Carbendazim+mancozeb (Sixer 75\% WP) & $\begin{array}{c}9.42 \\
(2.68)\end{array}$ & $\begin{array}{c}9.29 \\
(2.61)\end{array}$ & $\begin{array}{c}9.36 \\
(2.64)\end{array}$ & 75.93 & $\begin{array}{c}19.47 \\
(11.11)\end{array}$ & $\begin{array}{c}19.54 \\
(11.18)\end{array}$ & $\begin{array}{c}19.51 \\
(11.15)\end{array}$ & 60.25 \\
\hline 6. & MEMC (Emisan 6\% WP) & $\begin{array}{l}13.02 \\
(5.07)\end{array}$ & $\begin{array}{l}12.95 \\
(5.02)\end{array}$ & $\begin{array}{l}12.99 \\
(5.05)\end{array}$ & 53.97 & $\begin{array}{c}21.73 \\
(13.71)\end{array}$ & $\begin{array}{c}21.81 \\
(13.80)\end{array}$ & $\begin{array}{c}21.77 \\
(13.75)\end{array}$ & 50.98 \\
\hline 7. & Mancozeb (Diathane M-45, 75\% WP) & $\begin{array}{l}13.95 \\
(5.81)\end{array}$ & $\begin{array}{l}13.90 \\
(5.77)\end{array}$ & $\begin{array}{l}13.93 \\
(5.79)\end{array}$ & 47.22 & $\begin{array}{c}21.79 \\
(13.78)\end{array}$ & $\begin{array}{c}21.87 \\
(13.88)\end{array}$ & $\begin{array}{c}21.83 \\
(13.83)\end{array}$ & 50.70 \\
\hline 8. & Thiophanate methyl (Topsin M-75\% WP) & $\begin{array}{c}9.59 \\
(2.78)\end{array}$ & $\begin{array}{c}9.47 \\
(2.71)\end{array}$ & $\begin{array}{c}9.53 \\
(2.74)\end{array}$ & 75.02 & $\begin{array}{c}19.61 \\
(11.26)\end{array}$ & $\begin{array}{c}19.67 \\
(11.33)\end{array}$ & $\begin{array}{c}19.64 \\
(11.30)\end{array}$ & 59.71 \\
\hline 9. & Difenconazole (Score 25\% EC) & $\begin{array}{l}13.02 \\
(5.07)\end{array}$ & $\begin{array}{l}12.95 \\
(5.02)\end{array}$ & $\begin{array}{l}12.99 \\
(5.05)\end{array}$ & 53.97 & $\begin{array}{c}21.48 \\
(13.41)\end{array}$ & $\begin{array}{c}21.56 \\
(13.50)\end{array}$ & $\begin{array}{c}21.52 \\
(13.46)\end{array}$ & 52.01 \\
\hline 10. & Control (without treatment) & $\begin{array}{c}19.34 \\
(10.96)\end{array}$ & $\begin{array}{c}19.34 \\
(10.97)\end{array}$ & $\begin{array}{c}19.34 \\
(10.97)\end{array}$ & - & $\begin{array}{c}31.92 \\
(27.96)\end{array}$ & $\begin{array}{c}32.04 \\
(28.14)\end{array}$ & $\begin{array}{c}31.98 \\
(28.05)\end{array}$ & - \\
\hline \multicolumn{2}{|c|}{ SEm \pm} & 0.73 & 0.74 & 0.47 & - & 0.69 & 0.71 & 0.44 & - \\
\hline \multicolumn{2}{|c|}{$\mathrm{CD}(p=0.05)$} & 2.17 & 2.21 & 1.32 & - & 2.13 & 2.16 & 1.28 & - \\
\hline \multicolumn{2}{|c|}{ C.V. \% } & 10.19 & 10.44 & 10.31 & - & 5.68 & 6.12 & 5.90 & - \\
\hline
\end{tabular}

Data outside the paranthesis are ARCSIN transformed data; PDI-Percent disease intensity; PDC-Percent disease control

with thiophanate methyl (11.30\%), carbendazim (11.37\%) and captan (11.45\%). Next best treatment in order to merit was thiram (13.46\%) followed by difenconazole (13.75\%), MEMC (13.46\%), mancozeb (13.75\%) and kasugomycin (14.50\%). The highest disease was recorded in the control (28.05\%). Maximum control of Alternaria leaf spot was recorded in carbendazim + mancozeb $(60.25 \%)$ followed by thiophanate methyl $(59.71 \%)$, carbendazim $(59.47 \%)$, captan $(59.18 \%)$, thiram (52.01\%), difenconazole (52.01\%), MEMC (50.98\%), mancozeb (50.70\%) and kasugomycin (48.31\%).

\subsection{Anthracnose leaf spot at 35 DAS}

The pooled data of two years (Table 3 ) indicated that the PDI of anthracnose was significantly reduced in all the treatments over the control at 35 DAS. It was significantly lower in dry seed treatment with carbendazim + mancozeb $(0.81 \%)$ as compared to the rest but was statistically at par with thiophanate methyl (1.07\%). Next best treatment in order to merit was carbendazim (1.37\%) followed by captan (1.63\%), thiram (1.66\%), difenconazole (1.66\%), mancozeb $(1.81 \%)$,
MEMC (1.81\%). Kasugomycin (2.03\%) was found poor against anthracnose. The highest disease was recorded in the control (6.43\%). Maximum control of anthracnose was recorded in carbendazim + mancozeb $(87.40 \%)$ followed by thiophanate methyl (83.36\%), carbendazim (78.69\%), captan $(75.65 \%)$, thiram $(74.18 \%)$, difenconazole (74.18\%), MEMC (71.85\%), mancozeb (71.85\%) and kasugomycin (68.43\%).

\subsection{Anthracnose leaf spot at 65 DAS}

The pooled data of two years (Table 3 ) indicated that the PDI of anthracnose was significantly reduced in all the treatments over the control at 65 DAS. It was significantly lower in dry seed treatment with carbendazim + mancozeb (6.71\%) as compared to the rest but was statistically at par with thiophanate methyl (7.34\%), carbendazim (7.38\%) and captan (8.12\%). Next best treatment in order to merit was thiram (8.75\%), difenconazole (9.05\%), mancozeb (9.28\%), MEMC (9.76\%), and kasugomycin (10.32\%). Maximum control of anthracnose was recorded in carbendazim + mancozeb $(66.85 \%)$ followed by thiophanate methyl $(63.74 \%)$, carbendazim $(63.54 \%)$, captan $(59.88 \%)$, 


\begin{tabular}{|c|c|c|c|c|c|c|c|c|c|}
\hline \multirow{3}{*}{$\begin{array}{l}\text { Sr. } \\
\text { No. }\end{array}$} & \multirow[t]{3}{*}{ Treatment } & \multicolumn{8}{|c|}{ PDI ( Anthracnose: C. capsici) } \\
\hline & & \multicolumn{4}{|c|}{35 DAS } & \multicolumn{4}{|c|}{65 DAS } \\
\hline & & $\begin{array}{l}\text { First } \\
\text { year }\end{array}$ & $\begin{array}{l}\text { Second } \\
\text { year }\end{array}$ & Pooled & PDC & $\begin{array}{l}\text { First } \\
\text { year }\end{array}$ & $\begin{array}{c}\text { Second } \\
\text { year }\end{array}$ & Pooled & PDC \\
\hline 1. & Carbendazim (Bavistin 50\% WP) & $\begin{array}{c}6.82 \\
(1.41)\end{array}$ & $\begin{array}{c}6.60 \\
(1.32)\end{array}$ & $\begin{array}{c}6.71 \\
(1.37)\end{array}$ & 78.69 & $\begin{array}{l}15.85 \\
(7.46)\end{array}$ & $\begin{array}{l}15.67 \\
(7.30)\end{array}$ & $\begin{array}{l}15.76 \\
(7.38)\end{array}$ & 63.54 \\
\hline 2. & Kasugomycin (Kasugomycin 12\% WP) & $\begin{array}{c}8.28 \\
(2.07)\end{array}$ & $\begin{array}{c}8.12 \\
(1.99)\end{array}$ & $\begin{array}{c}8.20 \\
(2.03)\end{array}$ & 68.43 & $\begin{array}{c}18.83 \\
(10.41)\end{array}$ & $\begin{array}{c}18.65 \\
(10.22)\end{array}$ & $\begin{array}{c}18.74 \\
(10.32)\end{array}$ & 49.01 \\
\hline 3. & Thiram (Thirum 75\% WP) & $\begin{array}{c}7.50 \\
(1.70)\end{array}$ & $\begin{array}{c}7.31 \\
(1.62)\end{array}$ & $\begin{array}{c}7.41 \\
(1.66)\end{array}$ & 74.18 & $\begin{array}{l}17.30 \\
(8.84)\end{array}$ & $\begin{array}{l}17.12 \\
(8.67)\end{array}$ & $\begin{array}{l}17.21 \\
(8.75)\end{array}$ & 56.77 \\
\hline 4. & Captan (Captan 75\% WP) & $\begin{array}{c}7.43 \\
(1.67)\end{array}$ & $\begin{array}{c}7.22 \\
(1.58)\end{array}$ & $\begin{array}{c}7.32 \\
(1.63)\end{array}$ & 74.65 & $\begin{array}{l}16.65 \\
(8.21)\end{array}$ & $\begin{array}{l}16.47 \\
(8.04)\end{array}$ & $\begin{array}{l}16.56 \\
(8.12)\end{array}$ & 59.88 \\
\hline 5. & Carbendazim+mancozeb (Sixer 75\% WP) & $\begin{array}{c}5.30 \\
(0.85)\end{array}$ & $\begin{array}{c}5.00 \\
(0.76)\end{array}$ & $\begin{array}{c}5.15 \\
(0.81)\end{array}$ & 87.40 & $\begin{array}{l}15.10 \\
(6.79)\end{array}$ & $\begin{array}{l}14.92 \\
(6.63)\end{array}$ & $\begin{array}{l}15.01 \\
(6.71)\end{array}$ & 66.85 \\
\hline 6. & MEMC (Emisan 6\% WP) & $\begin{array}{c}7.73 \\
(1.81)\end{array}$ & $\begin{array}{c}7.73 \\
(1.81)\end{array}$ & $\begin{array}{c}7.73 \\
(1.81)\end{array}$ & 71.85 & $\begin{array}{l}17.82 \\
(9.37)\end{array}$ & $\begin{array}{l}17.64 \\
(9.19)\end{array}$ & $\begin{array}{l}17.73 \\
(9.28)\end{array}$ & 54.15 \\
\hline 7. & Mancozeb (Diathane M-45, 75\% WP) & $\begin{array}{c}7.82 \\
(1.85)\end{array}$ & $\begin{array}{c}7.65 \\
(1.77)\end{array}$ & $\begin{array}{c}7.73 \\
(1.81)\end{array}$ & 71.85 & $\begin{array}{l}18.29 \\
(9.85)\end{array}$ & $\begin{array}{l}18.11 \\
(9.67)\end{array}$ & $\begin{array}{l}18.20 \\
(9.76)\end{array}$ & 51.78 \\
\hline 8. & Thiophanate methyl (Topsin M-75\% WP) & $\begin{array}{c}6.05 \\
(1.11)\end{array}$ & $\begin{array}{c}5.80 \\
(1.02)\end{array}$ & $\begin{array}{c}5.92 \\
(1.07)\end{array}$ & 83.36 & $\begin{array}{l}15.81 \\
(7.42)\end{array}$ & $\begin{array}{l}15.63 \\
(7.26)\end{array}$ & $\begin{array}{l}15.72 \\
(7.34)\end{array}$ & 63.74 \\
\hline 9. & Difenconazole (Score 25\% EC) & $\begin{array}{c}7.50 \\
(1.70)\end{array}$ & $\begin{array}{c}7.31 \\
(1.62)\end{array}$ & $\begin{array}{c}7.41 \\
(1.66)\end{array}$ & 74.18 & $\begin{array}{l}17.60 \\
(9.14)\end{array}$ & $\begin{array}{l}17.42 \\
(8.96)\end{array}$ & $\begin{array}{l}17.51 \\
(9.05)\end{array}$ & 55.29 \\
\hline 10. & Control (without treatment) & $\begin{array}{l}14.70 \\
(6.44)\end{array}$ & $\begin{array}{l}14.67 \\
(6.41)\end{array}$ & $\begin{array}{l}14.69 \\
(6.43)\end{array}$ & - & $\begin{array}{c}26.82 \\
(20.36)\end{array}$ & $\begin{array}{c}26.64 \\
(20.11)\end{array}$ & $\begin{array}{c}26.73 \\
(20.24)\end{array}$ & - \\
\hline \multicolumn{2}{|c|}{ SEm \pm} & 0.66 & 0.68 & 0.42 & - & 1.07 & 1.06 & 0.67 & - \\
\hline \multicolumn{2}{|c|}{$\mathrm{CD}(p=0.05)$} & 1.98 & 2.04 & 1.21 & - & 3.19 & 3.15 & 1. 93 & - \\
\hline \multicolumn{2}{|c|}{ C.V. \% } & 12.86 & 13.92 & 13.39 & - & 10.42 & 10.34 & 10.38 & - \\
\hline
\end{tabular}

Data outside the paranthesis are ARCSIN transformed data; PDI-Percent disease intensity; PDC-Percent disease control

thiram (56.77\%), difenconazole (55.29\%), MEMC (54.15\%), mancozeb (51.78\%) and kasugomycin (49.01\%).

The present results are more or less similar with earlier workers. Fungicidal seed treatment with Agallol, Captan or Thirum (Suhag, 1975) and Bavistin, Thiram and Diathane M-45 (Zote and Mayee, 1982) proved better in promoting the germination and seedling vigour of moong bean seeds. Reddy et al. (1992) showed that fungicidal seed dressing with flutolanil, thiophanate-methyl or carbendazim effectively controlled damping-off of Vigna radiata, while Mancozeb and zineb were the least effective. Moreover to these results, Dash and Narain (1996) also found that pre-treatment of seeds of $V$. radiata with carbendazim + thiram followed by thiram, quintozene, difolatan, mancozeb and carboxin eliminated seed mycoflora and improved seed germination considerably for most of the test crops. Agrawal et al. (1989) noticed that seed treatment with carbendazim reduced seed and seedling mortality caused by M. phaseolina in V. radiata. The initial leaf blight phase of the disease was also found less. Bidari et al.
(1992) observed that seed treatment with carbendazim gave the best control of seed borne pathogens by reducing seed rot, seedling mortality and resulting in the highest yield of the $V$. radiata crop. Good control was also obtained in Phenyl mercury acetate, Dithane $\mathrm{M}$ and Thiram at $2 \mathrm{~g} \mathrm{~kg}^{-1}$ seed under field condition. Khan and Khan (2006) also reported that seed treatment with $0.10 \%$ Topsin $\mathrm{M}$ showed significantly higher reduction in Macrophomina leaf spot incidence at 45, 55 and 70 days after sowing and recorded the highest increase in green gram yield (46.8\%) followed by benomyl (32.9\%) and carbendazim (31.40\%). The control of anthracnose and Alternaria leaf spot by fungicidal seed treatment carried out here is the first exempt but the work done in other crops was in similar line with the present study. Suradkar (2010) found that maximum control of anthracnose caused by $C$. capsici was obtained in seed treatment of carbendazim (81.57\%) followed by thiram (76.31\%), MEMC (64.47\%) and mancozeb (63.15\%) along with better increased in seed germination in blackgram. Laxminarayanarao (2006) found that seed dressing with 
carbendazim + iprodione was most effective in eliminating seed borne infections of Alternaria sp. in sunflower causing Alternaria blight.

\section{Conclusion}

Dry seed treatmemt with either mixed formulation of carbendazim+mancozeb or thiophanate methyl or carbendazim @2.5 g kg-1 of seeds is recommended for the management of seed borne diseases of green gram viz., leaf blight (M. phaseolina), leaf spot (A. alternata) and anthracnose (C. capsici).

\section{References}

Agrawal, S.C., 1989. Diseases of green gram and black gram. International Book distributors, 1-2, 5, 28-29, 3233,159,181,269.

Agrawal, S.C., Sushma Nema, Nema, S., 1989. Effect of carbendazim on Macrophomina leaf blight of black gram and green gram. Indian Journal of Plant Protection 17, 147-149.

Bidari, V.B., Dayanand, M., Anahosur, K.H., Mannur, D.M., 1992. Field evaluation of seed treating fungicides against seed rot in greengram. Indian Journal of Pulses Research 5, 94-96.

Bilgrami, K.S., Sinha, R.K., Prasad, T., 1978. Effect of fungal flora on the seed contents of moong. Indian Phytopathology 31, 476-479.

Dash, S.K., Narain, A., 1996. Efficacy of selected fungicides on seed-borne fungi and on percentage of germination of diseased seeds of crops. Crop Research-Hisar 11, 207-211.

John, M.P., 1991. The mung bean. Pub: Oxford and IBH Publishing Co. Pvt. Ltd.66 Janpath, New Delhi, 126-127.

Khan, A.A., Khan, R.U., 2006. Management of macrophomina leaf spot of Vigna radiata by fungicides. Annals-of-PlantProtection-Sciences 14, 258-259.

Kumar, S.M., Khare, M.N., Srivastav, S.K., 1969. Macrophomina leaf spot of urd (Phaseolus mungo). Some observations on disease resistance. Mysore Journal of Agricultural Sciences 3, 472-474.

Laxminarayanarao, M.S., 2006. Studies on seed-borne fungal diseases of sunflower and their management with special reference to the alternaria blight. Ph.D. Thesis submitted to the University of Agricultural Sciences,
Dharwad.

Mathur, R. L., Mathur, B.N., Sharma, B.S., 1972. Relative efficacy of fungicides for the control of Alternaria cyamopsidis causing leaf spot of guar (Cyamopsidis tetragonaloba). Indian J.ournal of Mycology and Plant Pathology 2, 80-81.

Patel, J.P., 2003. Investigation on leaf spot of green gram (Phaseolus aureus Roxb) caused by Alternaria alternata (Fr.) Keissler under south Gujarat condition. M.Sc. (Agri.) Thesis submitted to N.A.U., Navsari.

Rajkumar, Mukhopadhyay, A.N., 1986. Field evaluation of urd bean germplasm lines against Colletotrichum capsici. Indian Journal of Mycology and Plant Pathology 17, 66.

Rangaswami, G., Prasad, N., 1960. A new seedling blight of Phaseolus aureus Roxb. And Phaseolus mungo L. Indian Phytopathology 12, 184-185.

Rayen, G.F., 1961. Seedling albinism induced by an extract of Alternaria tenuis. Science 134, 833-834.

Reddy, K.S., Rao, K.C., Reddy, M.S., 1992. Evaluation of some new fungicides against Rhizoctonia solani Kuhn, the incitant of damping-off of mungbean. Indian Journal of Plant Protection 20, 37-42.

Singh, H.B., Joshi, B.S., Thomas, T.A., 1970. In Kachroo, P. (ed.) pulse crops of India, New Delhi: ICAR.

Sinha, R.K., Prasad, T., 1981. Effect of fungal metabolites on seed germination, microbial association and seedling growth of Mung. Indian Phytopatholgy 34, 515-517.

Suhag, L.S., 1975. Fungal flora of Moong (Phaseolus aureus) seeds: pathology and control. Indian Journal of Mycology and Plant Pathology 5, 165-168.

Suradkar, S.R., 2010. Investigation on anthracnose [Colletotrichum capsici (Syd.) Butler and Bisby] of Blackgram (Vigna mungo L. Hepper) under south Gujarat condition. M.Sc. (Agri.) thesis submitted to NAU, Navsari.

Tan, S.Y., 1973. Food Industries (Taiwan) 5, 20-26.

Tandel, D.H., 2004. Epidemiology and management of leaf blight of green gram (Phaseolus aureus Roxb) caused by Macrophomina phaseolina (Tassi) Goid. M.Sc. (Agri.) thesis submitted to NAU, Navsari.

Tsou, C.S., Hsu, M.S., Tan, S., Park, H.G., 1979. Acta Horticulture 93, 279-287.

Zote, K.K., Mayee, C.D., 1982. Influence of fungicidal seed treatment during storage on seed borne fungi of mung bean. Pesticides, 10-12. 